\title{
Knowledge of Postpartum Women About Modern Contraceptive Methods and Attitudes Towards its Utilization in Debre Tabor Town, Northwest Ethiopia: A Community-Based Cross-Sectional Study
}

Eden Taye ( $\nabla$ edbishaw16@gmail.com ) University Of Gondar

Dawit Mekonnen

University Of Gondar

Tibeb Debele

University Of Gondar

Agumas Tiguh

University Of Gondar

Azmeraw Kebede

University Of Gondar

\section{Research Article}

Keywords: Attitude, Ethiopia, knowledge, modern contraceptives

Posted Date: December 29th, 2020

DOI: https://doi.org/10.21203/rs.3.rs-130623/v1

License: (c) (i) This work is licensed under a Creative Commons Attribution 4.0 International License.

Read Full License 


\section{Abstract}

Background: The postpartum period is a crucial time to prevent short inter-pregnancy interval and unwanted pregnancies. Despite it is the most ideal period to create awareness regarding knowledge and attitudes of postpartum mothers about contraceptive methods, it is the most neglected part of the continuum of care. Therefore, this study aimed to assess knowledge of postpartum women about modern contraceptive methods and attitudes towards its utilization in Debre Tabor town, northwest Ethiopia, 2018.

Methods: A community-based cross-sectional study was conducted among 552 postpartum mothers at Debre Tabor town from September 20 to October 20, 2018. A cluster sampling technique was used to select the study participants. A pre-tested structured questionnaire was used to collect the data. Data were entered into Epi info version 7 and analyzed by SPSS version 20 software. Multivariable logistic regression model was fitted to identify associated factors.

Results: The overall prevalence of good knowledge and favorable attitude towards modern contraceptive methods was $57.6 \%$ (95\% Cl: 53.3\%, 61.6\%) and 40.6\% (95\% Cl: 36.8, 44.6) respectively. Higher education [AOR: 2.15 (95\% Cl: 1.36, 3.4)], ever had a discussion with their husband about contraception [AOR: 2.15 (95\% Cl: 1.48, 3.14)] and ever used modern contraceptive methods [AOR: 1.87 (95\% Cl: 1.15, 3.03)] were found to be significantly associated with good knowledge of postpartum modern contraceptive methods. Besides, maternal age of 25-34-year-old ( $A O R=0.42 ; 95 \% \mathrm{Cl}: 0.27,0.65)$, having prior information about contraceptive methods (AOR $=1.73 ; 95 \% \mathrm{Cl}: 1.19,2.53)$ and good knowledge of postpartum modern contraceptive methods were associated with the attitude of mothers towards modern contraceptive methods.

Conclusion: More than half of the respondents had good knowledge about modern contraceptive methods. But, mother's favorable attitude towards its utilization is lower compared to other studies. Mother's higher educational status, ever discussed about contraceptive methods with their husband, and ever used contraceptive methods were the factors significantly associated with knowledge of postpartum modern contraceptive methods. Furthermore, adequate knowledge of postpartum modern contraceptive methods, age group of 25-34 years old and having prior information about contraceptive methods were the factors that significantly associated with the attitude of women.

\section{Introduction}

Family planning (FP) is the ability of individuals and couples to anticipate and attain their desired number of children and the spacing and timing of their births which is achieved through the use of contraceptive methods. It is also a way of thinking and living that is adopted voluntarily upon the basis of knowledge, attitude, and responsible decisions by individuals and couples (1).

Family planning is a means of promoting the health of women and families and part of a strategy to reduce the high maternal, infant and child mortality. Besides, it is also the most effective measure to 
control high population growth (2). For this reason, information about FP should be made available, and should actively promote access to FP services for all individuals desiring them (1).

Family planning is a priority area in the to-do lists of the government of Ethiopia; because it is one of the strategies of the government to improve maternal and child health. The newly launched health service development plan (HSDP) IV and the SDG (sustainable development goal) 7, are aiming at expanding quality FP services thereby improve the health of mothers, neonates, children, adolescents, and youths (3).

Lack of adequate data in the populations on knowledge and attitudes about modern contraceptives impede the effort to improve the practice of modern contraceptive methods (4). Information about FP should be made available, and should actively promote access to FP services for all individuals desiring them (1). Studying knowledge and attitudes of postpartum mothers tell us what they know and how they feel about modern contraceptives.

In Ethiopia, the prevalence of children born less than two years after their siblings is $22 \%$ (5). Short birth intervals are associated with harmful outcomes for both newborns and their mothers, such as preterm birth, low birth weight, and death (6-8). The postpartum period is the most crucial time to maintain the ultimate health of the women and her newborn. Also, it is the time of opportunity to provide contraceptive methods thereby decreasing the risk of short inter-pregnancy interval and unwanted pregnancies. Assessing postpartum mother's knowledge and attitude towards modern contraceptives is a way to improve its utilization which prevents mothers and their families from the consequences of shortly spaced pregnancies.

The majority of $\mathrm{MNCH}$ services are conducted before and within the first six months of the postpartum period. These $\mathrm{MNCH}$ services are an opportunity to build the mother's attitude and improve their knowledge of modern contraceptive methods. At the time of antenatal care (ANC), labor and delivery, postnatal care, and immunization service, women may get more information about modern contraceptive methods. So, it is an opportunity to assess and realize the knowledge and attitudes of postpartum mothers to improve the utilization of modern contraceptives.

Several studies assessed the utilization and factors affecting contraceptive methods $(9,10)$. However, only a few assessed mother's knowledge of postpartum modern contraceptive methods and attitudes towards its utilization. Studies elsewhere in Ethiopia revealed that mother's knowledge of postpartum modern contraceptive methods was $60.1 \%$ in East Gojjam, northwest Ethiopia (11), and $68.2 \%$ in eastern Ethiopia (12). Besides, the attitude of women towards postpartum modern contraceptive methods was $79.5 \%$ in Oromia region Ethiopia (13), and $51 \%$ in eastern Ethiopia (12). Though these studies assessed the knowledge of postpartum mothers about modern contraceptives, the present study adds questions like when a postpartum mother could start contraceptive methods, time of pregnancy after discontinuation of contraception and others in common. 
Increasing women's knowledge of the postpartum modern contraceptive method is the core to decrease unwanted pregnancy and short inter-pregnancy intervals thus improving the women's quality of life. Therefore, this study aimed to determine knowledge of postpartum modern contraceptive methods and attitudes towards its utilization in Debre Tabor town, northwest Ethiopia.

\section{Methods And Materials}

\section{Study Design, Setting and Period}

A community-based cross-sectional study was conducted from September 20 to October 20, 2018 in Debre Tabor town which is the capital city of South Gondar zone Amhara Regional State, Northwest Ethiopia. The town is located 666 kilometers northwest of Addis Ababa. Based on the 2007 National Census conducted by the Central Statistical Agency of Ethiopia (CSAE), projection the town has a total population of 55,596 of whom $27,644(49.7 \%)$ are men and $27,952(50.3 \%)$ are women. $96.72 \%$ of the population of the town is Orthodox Christians, and $2.54 \%$ is Muslim (14). The town has one referral hospital, three health centers, and six health posts serving the community.

\section{Study Population}

All women who gave birth in the past six to twelve months in the selected clusters during the study period were included. Mothers who were critically ill throughout the data collection period were excluded.

\section{Sample Size Determination and Sampling Procedures}

The sample size ( $\mathrm{n}$ ) which was required for this study was determined by using a single population proportions formula by considering the assumptions: postpartum mothers who had good knowledge was $68.2 \%(12)$, a margin of error $(d)=5 \%$, and a $95 \%$ level confidence.

$$
\mathrm{n}=\frac{(Z \alpha / 2)^{2} p(1-p)}{d^{2}}=\mathrm{n}=\frac{(1.96)^{2} * 0.682(1-0.682)}{(0.05) 2}=334
$$

Where $\mathrm{n}=$ required sample size, $\mathrm{a}=$ level of significant, $\mathrm{z}$ = standard normal distribution curve value for $95 \%$ confidence level $=1.96, p=$ proportion of mother's knowledge about postpartum modern contraceptive methods, $d=$ margin of error.

The final sample size was 552 including the design effect of 1.5 and a $10 \%$ non-response rate. Design effect of 1.5 was used in case of cost minimization rather than 2. Debre Tabor town has six urban "kebeles" (which are the smallest administrative unit in Ethiopia) and three "kebeles" were selected randomly (kebele 1, kebele 2, and kebele 5). A house-to-house visit was carried out in the selected clusters to identify eligible mothers. All eligible mothers in the selected clusters were interviewed with the assumptions of homogeneity of participants in Debre Tabor town.

\section{Operational Definitions}




\section{Extended postpartum period}

the period between six to twelve months of delivery (15).

\section{Good knowledge}

Based on the summative score of eleven variables intended to assess knowledge of mothers, a mother answered a score greater or equal to the mean value (six and above variables) was considered as knowledgeable (12).

\section{Poor knowledge}

Based on the summative score of eleven variables considered to assess knowledge of mothers, a mother answered a score less than the mean value ( five and below variables) was considered as not knowledgeable (12).

\section{Favorable attitude}

Based on the summative score of eight variables designed to assess the attitude of mothers, a mother answered a score above and equal to the mean value (four and above variables) was considered as having a favorable attitude.

\section{Unfavorable attitude}

Based on the summative score of eight variables designed to assess the attitude of mothers, a mother answered score less than the mean value (three and les $s$ variables) was considered as having unfavorable attitude.

\section{Data Collection Tools, Procedures and Quality Control}

Data was collected using face to face interviewer-administered questionnaire. Two fourth-year midwifery students and one BSc in midwifery holders were recruited for data collection and supervision respectively. The questionnaires were prepared in English and translated into Amharic and translated back to English to keep the consistency of the questionnaire. We did a pre-test on $28(5 \%)$ of the sample at Woreta town. One day training was given about the overall data collection process for both the data collectors and the supervisor. During the data collection period, questionnaire was checked for completeness daily by the supervisors, and on spot corrective measures was taken.

\section{Data Processing and Analysis}

The data were entered into EPI Info version 7 and exported to SPSS version 20 software for analysis. Binary logistic regression was used to identify factors associated with knowledge and attitudes of modern contraceptive methods. Variables having with a p-value of less than or equal to 0.20 was fitted in the multivariable logistic regression models for controlling the possible effect of confounders and the variables which have independent association with knowledge and attitudes of postpartum mothers 
about modern contraceptive methods were identified based on the odds ratio with $95 \% \mathrm{Cl}$ and $p$-value less than or equal to 0.05 were considered to determine statically significant association.

\section{Results}

\section{Socio-Demographic Characteristics}

A total of 552 postpartum mothers were included in the study. The mean age of the participants was 27.59 years ( $S D \pm 4.82$ ). Most $(95.1 \%$ ) of the mothers were married and $96.7 \%$ of the mothers were orthodox Christians. Regarding the educational status, 344 (62.3\%) of mothers were secondary education and above. Among respondents, 336 (60.9\%) were a housewife by occupation (Table 1). 
Table 1

Socio-Demographic Characteristics of Postpartum Mothers in Debre Tabor Town, South Gondar Zone, Northwest Ethiopia, 2018 ( $n=552)$

\begin{tabular}{|c|c|c|}
\hline Variables & Frequency & Percent (\%) \\
\hline \multicolumn{3}{|l|}{ Age } \\
\hline $18-24$ & 132 & 23.9 \\
\hline $25-34$ & 353 & 63.9 \\
\hline $35-45$ & 67 & 12.1 \\
\hline \multicolumn{3}{|l|}{ Marital status } \\
\hline Married & 525 & 95.1 \\
\hline Single & 11 & 2 \\
\hline Divorced & 14 & 2.5 \\
\hline Widowed & 2 & 0.4 \\
\hline \multicolumn{3}{|l|}{ Religion } \\
\hline orthodox & 534 & 96.7 \\
\hline Muslim & 15 & 2.7 \\
\hline Protestant & 3 & 0.5 \\
\hline \multicolumn{3}{|l|}{ Level of education } \\
\hline Illiterate & 115 & 20.8 \\
\hline Primary education & 93 & 16.8 \\
\hline High school and above & 344 & 62.3 \\
\hline \multicolumn{3}{|l|}{ Occupation } \\
\hline Housewife & 336 & 60.9 \\
\hline government and private employed & 113 & 20.5 \\
\hline Laborer & 6 & 1.1 \\
\hline self-employed & 61 & 11.1 \\
\hline Others (farmers and student) & 36 & 6.5 \\
\hline \multicolumn{3}{|l|}{ Partners educational status $(n=538)$} \\
\hline Illiterate & 102 & 19 \\
\hline Primary education & 72 & 13.4 \\
\hline
\end{tabular}




\begin{tabular}{|c|c|c|}
\hline Variables & Frequency & Percent (\%) \\
\hline High school and above & 364 & 67.7 \\
\hline \multicolumn{3}{|l|}{ Partners occupation ( $n=538$ ) } \\
\hline Gov't employed \& private employed & 265 & 49.3 \\
\hline Daily laborer & 59 & 11 \\
\hline self-employed & 187 & 34.8 \\
\hline Others (farmer and student) & 27 & 5 \\
\hline
\end{tabular}

About 537 (97.3\%) of the mothers were delivered at health institutions for their most recent delivery. More than three-fourths $(76.1 \%)$ of deliveries were spontaneous delivery. About 237 (42.9\%) of mothers have discussed with their husbands about FP methods. Most of the mothers 537 (97.6\%) had ANC follow up and more than four-fifths (86.1\%) of women had four and above visits. More than half $(56.9 \%)$ of the mothers had PNC visit of whom 163 (51.9\%) got information about modern contraceptives in the postpartum period (Table 2). 
Table 2

Reproductive and Maternity Health Service-Related Characteristics of Mother at Debre Tabor Town Northwest Ethiopia, $2018(n=552)$

\begin{tabular}{|c|c|c|}
\hline Variables & Frequency & Percent (\%) \\
\hline \multicolumn{3}{|l|}{ Parity } \\
\hline $1-2$ & 411 & 74.5 \\
\hline $3-4$ & 124 & 22.5 \\
\hline$>=5$ & 17 & 3.1 \\
\hline \multicolumn{3}{|l|}{ Place of delivery } \\
\hline Home delivery & 15 & 2.7 \\
\hline Institutional delivery & 537 & 97.3 \\
\hline \multicolumn{3}{|l|}{ Mode of delivery } \\
\hline Spontaneous vaginal delivery & 420 & 76.1 \\
\hline Caesarean delivery & 55 & 10 \\
\hline Instrumental delivery & 77 & 13.9 \\
\hline \multicolumn{3}{|c|}{ Intension to have another child for the future } \\
\hline No & 121 & 21.9 \\
\hline Yes & 431 & 78.1 \\
\hline \multicolumn{3}{|c|}{ Ever discussed with their husband about FP } \\
\hline No & 315 & 57.1 \\
\hline Yes & 237 & 42.9 \\
\hline \multicolumn{3}{|l|}{ Do you have ANC visit } \\
\hline No & 13 & 2.4 \\
\hline Yes & 539 & 97.6 \\
\hline \multicolumn{3}{|l|}{ Number of ANC visit $(n=539)$} \\
\hline$\geq 4$ times & 464 & 86.1 \\
\hline$<4$ times & 75 & 13.9 \\
\hline \multicolumn{3}{|c|}{ Get information on FP during ANC $(n=539)$} \\
\hline No & 244 & 45.3 \\
\hline Yes & 295 & 54.7 \\
\hline
\end{tabular}




\begin{tabular}{|c|c|c|}
\hline Variables & Frequency & Percent (\%) \\
\hline \multicolumn{3}{|c|}{ Attend PNC } \\
\hline No & 238 & 43.1 \\
\hline Yes & 314 & 56.9 \\
\hline \multicolumn{3}{|c|}{ Get information about FP on PNC $(n=314)$} \\
\hline No & 151 & 48.1 \\
\hline Yes & 163 & 51.9 \\
\hline \multicolumn{3}{|c|}{$\begin{array}{l}\text { Get information about family planning before } \\
\text { the delivery of last child }\end{array}$} \\
\hline No & 310 & 56.2 \\
\hline Yes & 242 & 43.8 \\
\hline \multicolumn{3}{|c|}{$\begin{array}{l}\text { After delivery of the last child do you get information } \\
\text { about family planning }\end{array}$} \\
\hline No & 342 & 62 \\
\hline Yes & 210 & 38 \\
\hline \multicolumn{3}{|c|}{ History of modern contraceptive before the delivery of last child } \\
\hline No & 97 & 17.6 \\
\hline Yes & 455 & 82.4 \\
\hline
\end{tabular}

Among the respondents, 318 (57.6\%) had good knowledge of postpartum modern contraceptive methods. Most of the participants 482 (87.3\%) were ever heard about various methods of contraceptives used for postpartum mothers. Nearly two-thirds $(74.6 \%)$ of the mothers knew that a woman can pregnant whilst breastfeeding. Above half of the respondents knew postpartum women can start contraceptives before the beginning of menstruation. One-fourths (26.8\%) of the mothers knew that IUCD can be used by breastfeeding mothers. Nearly half $(47.1 \%)$ of the participants knew that after permanent contraceptive a woman cannot become pregnant (Table 3$)$. Injectables (84.4\%), pills (74.3\%), and implants (60.3\%) were the most known contraceptive methods by the study participants (Fig. 1). 
Table 3

Knowledge of Women in the Postpartum Period on Modern Contraceptives in Debre Tabor Town, Northwest Ethiopia, $2018(n=552)$

\begin{tabular}{|c|c|c|}
\hline Variables & Frequency & Percent \\
\hline \multicolumn{3}{|c|}{ Know family planning methods used for postpartum mothers } \\
\hline Yes & 482 & 87.3 \\
\hline No & 70 & 12.7 \\
\hline \multicolumn{3}{|c|}{ Know mother become pregnant whilst breastfeeding } \\
\hline Yes & 412 & 74.6 \\
\hline No & 140 & 25.4 \\
\hline \multicolumn{3}{|c|}{ Know a woman start contraception before the menstruation begins? } \\
\hline Yes & 333 & 60.3 \\
\hline No & 219 & 39.7 \\
\hline \multicolumn{3}{|c|}{ Know IUCD be used to prevent pregnancy after delivery } \\
\hline Yes & 234 & 42.4 \\
\hline No & 318 & 57.6 \\
\hline \multicolumn{3}{|c|}{$\begin{array}{l}\text { If IUCD is used to prevent pregnancy after delivery, when to start it? } \\
(n=234)\end{array}$} \\
\hline After delivery within 48 hours & 42 & 17.9 \\
\hline From 4-6 weeks. & 28 & 12 \\
\hline From 6 weeks- 6 months & 89 & 38 \\
\hline From 7 months- 1 year & 12 & 5.1 \\
\hline I don't know & 63 & 26.9 \\
\hline \multicolumn{3}{|c|}{ Know IUCD used during breast feeding period } \\
\hline Yes & 148 & 26.8 \\
\hline No & 404 & 73.2 \\
\hline \multicolumn{3}{|c|}{ Know after IUCD removal a woman can become pregnant immediately } \\
\hline Yes & 189 & 34.2 \\
\hline No & 363 & 65.8 \\
\hline
\end{tabular}




\begin{tabular}{|c|c|c|}
\hline Variables & Frequency & Percent \\
\hline Yes & 344 & 62.3 \\
\hline No & 208 & 37.7 \\
\hline \multicolumn{3}{|c|}{ If implants used to prevent pregnancy after delivery when to start it? $(n=344)$} \\
\hline from delivery - 6 weeks & 163 & 47.4 \\
\hline from 7 week - 6months & 84 & 24.4 \\
\hline from 7 months -1 year & 46 & 13.4 \\
\hline I don't know & 51 & 14.8 \\
\hline \multicolumn{3}{|c|}{ Know after removal of implants a woman can become pregnant immediately } \\
\hline Yes & 295 & 53.4 \\
\hline No & 257 & 46.6 \\
\hline \multicolumn{3}{|c|}{ Know after permanent contraceptive a woman can't become pregnant } \\
\hline Yes & 260 & 47.1 \\
\hline No & 292 & 52.9 \\
\hline \multicolumn{3}{|c|}{ Know a woman during breast feeding period can use COC } \\
\hline Yes & 223 & 40.4 \\
\hline No & 329 & 59.6 \\
\hline \multicolumn{3}{|c|}{ If $\mathrm{COC}$ is used for during breast feeding when to start it? $(\mathrm{N}=223)$} \\
\hline From delivery - 6 weeks & 106 & 47.6 \\
\hline From 7 week - 5 months & 72 & 32.3 \\
\hline from 6 months -1 year & 21 & 9.4 \\
\hline I don't know & 24 & 10.8 \\
\hline \multicolumn{3}{|c|}{ Know Injectables (DEPO) be used to prevent pregnancy after delivery } \\
\hline Yes & 449 & 81.3 \\
\hline No & 103 & 18.7 \\
\hline \multicolumn{3}{|c|}{ If Injectables used after delivery when to start after delivery $(n=449)$} \\
\hline Immediately after delivery & 13 & 2.9 \\
\hline From 48 hours -6 days & 4 & .9 \\
\hline From 7 days -3 weeks & 22 & 4.9 \\
\hline
\end{tabular}




\begin{tabular}{|lll|}
\hline Variables & Frequency & Percent \\
\hline 3 weeks-6months & 303 & 67.5 \\
\hline Above 6 months & 80 & 17.8 \\
\hline I don't know & 27 & 6 \\
\hline Overall knowledge & & \\
\hline Poor Knowledge & 234 & 42.4 \\
\hline Good Knowledge & 318 & 57.6 \\
\hline
\end{tabular}

\section{Mother's Attitude of Postpartum Modern Contraceptive Methods}

Among the respondents, $224(40.6 \%)$ had a favorable attitude towards postpartum modern contraceptive methods utilization (Table 4). 
Table 4

Attitudes of Women in the Postpartum Period on Modern Contraceptive Methods in Debre Tabor Town, Northwest Ethiopia, $2018(\mathrm{n}=552)$

\begin{tabular}{|c|c|c|c|c|c|}
\hline Variables & $\begin{array}{l}\text { Strongly } \\
\text { disagree }\end{array}$ & Disagree & Neutral & Agree & $\begin{array}{l}\text { Strongly } \\
\text { agree }\end{array}$ \\
\hline $\begin{array}{l}\text { Without family planning Postpartum } \\
\text { woman can pregnant within } 6 \text { weeks. }\end{array}$ & $\begin{array}{l}52 \\
(9.4 \%)\end{array}$ & $147(26.6 \%)$ & $\begin{array}{l}75 \\
(13.6 \%)\end{array}$ & $\begin{array}{l}235 \\
(42.6 \%)\end{array}$ & $\begin{array}{l}43 \\
(7.8 \%)\end{array}$ \\
\hline $\begin{array}{l}\text { After delivery if the menses is not } \\
\text { started I will not become pregnant }\end{array}$ & $\begin{array}{l}125 \\
(22.6 \%)\end{array}$ & 157(28.4\%) & $\begin{array}{l}35 \\
(6.3 \%)\end{array}$ & $\begin{array}{l}176 \\
(31.9 \%)\end{array}$ & $\begin{array}{l}59 \\
(10.7 \%)\end{array}$ \\
\hline $\begin{array}{l}\text { PPFP doesn't affect the health of my } \\
\text { child }\end{array}$ & $\begin{array}{l}20 \\
(3.6 \%)\end{array}$ & $107(19.4 \%)$ & $\begin{array}{l}116 \\
(21 \%)\end{array}$ & $\begin{array}{l}233 \\
(42.2 \%)\end{array}$ & $\begin{array}{l}76 \\
(13.8 \%)\end{array}$ \\
\hline $\begin{array}{l}\text { Postpartum family planning doesn't } \\
\text { help me to start sexual intercourse } \\
\text { immediately. }\end{array}$ & $\begin{array}{l}43 \\
(7.8 \%)\end{array}$ & $132(23.9 \%)$ & $\begin{array}{l}241 \\
(43.7 \%)\end{array}$ & $\begin{array}{l}81 \\
(14.7 \%)\end{array}$ & $\begin{array}{l}55 \\
(10 \%)\end{array}$ \\
\hline $\begin{array}{l}\text { Postpartum family planning can't } \\
\text { expose to urinary tract infection. }\end{array}$ & $11(2 \%)$ & 67 (12.1\%) & $\begin{array}{l}217 \\
(39.3 \%)\end{array}$ & $\begin{array}{l}150 \\
(27.2 \%)\end{array}$ & $\begin{array}{l}107 \\
(19.4 \%)\end{array}$ \\
\hline $\begin{array}{l}\text { IUCD doesn't affect any day to day } \\
\text { activity. }\end{array}$ & $7(1.3 \%$ & 53 (9.6\%) & $\begin{array}{l}286 \\
(51.8 \%)\end{array}$ & $\begin{array}{l}136 \\
(24.6 \%\end{array}$ & $\begin{array}{l}70 \\
(12.7 \%)\end{array}$ \\
\hline $\begin{array}{l}\text { Male sterilization doesn't affect the } \\
\text { desire of sexual activity. }\end{array}$ & $\begin{array}{l}27 \\
(4.9 \%)\end{array}$ & $55(10 \%)$ & $\begin{array}{l}312 \\
(56.5 \%)\end{array}$ & $\begin{array}{l}109 \\
(19.7 \%)\end{array}$ & $\begin{array}{l}49 \\
(8.9 \%)\end{array}$ \\
\hline $\begin{array}{l}\text { IUCD doesn't affect any sexual activity } \\
\text { and desire. }\end{array}$ & $33(6 \%)$ & 65 (11.8\%) & $\begin{array}{l}282 \\
(51.1 \%)\end{array}$ & $\begin{array}{l}111 \\
(20.1 \%)\end{array}$ & $\begin{array}{l}61 \\
(11.1 \%)\end{array}$ \\
\hline \multirow[t]{2}{*}{ Attitude } & \multicolumn{3}{|l|}{ Favorable } & \multicolumn{2}{|c|}{$224(40.6 \%)$} \\
\hline & \multicolumn{2}{|c|}{ Un -favorable } & & \multicolumn{2}{|c|}{328 (59.4\%) } \\
\hline
\end{tabular}

On the bivariable regression analysis, the factors found to be significantly associated with knowledge of mothers on modern contraceptive methods were: educational statuses of the mother, mothers ever discuss with their husband about contraceptive methods, attend PNC, having prior information about modern contraceptive methods after delivery of the last child, and history of modern contraceptive utilization before the delivery of the last child.

After controlling the effect of other variables, mothers educational status (high school and above) [AOR: 2.15 (95\% Cl: 1.36, 3.4)], ever discuss with their husband about contraceptive methods [AOR: 2.152 (95\% Cl: 1.48, 3.14)], and history of modern contraceptive utilization before the delivery of the last child [AOR: 1.87 (95\% Cl: 1.15, 3.03)] were found to be significantly associated with knowledge of modern contraceptive methods of postpartum mothers in multiple logistic regression analysis (Table 5). 
Table 5

Bivariable and Multivariable Logistic Regression Analysis of Factors Associated with Knowledge of Postpartum Mothers about Contraceptives in Debre Tabor Town, South Gondar Zone, Northwest Ethiopia, $2018(n=552)$

\begin{tabular}{|c|c|c|c|c|}
\hline \multirow[t]{2}{*}{ Variables } & \multicolumn{2}{|l|}{ Knowledge } & \multirow[t]{2}{*}{$\operatorname{COR}(95 \% \mathrm{Cl})$} & \multirow[t]{2}{*}{ AOR $(95 \% \mathrm{Cl})$} \\
\hline & $\begin{array}{l}\text { Good } \\
\text { knowledge }\end{array}$ & $\begin{array}{l}\text { poor } \\
\text { knowledge }\end{array}$ & & \\
\hline \multicolumn{5}{|l|}{ Marital status } \\
\hline Married & 306 & 219 & $1.747(.802,3.805)$ & $.839(.354,1.989)$ \\
\hline $\begin{array}{l}\text { Others (single, } \\
\text { divorced, widowed) }\end{array}$ & 12 & 15 & 1 & 1 \\
\hline \multicolumn{5}{|l|}{ Educational status } \\
\hline No formal education & 48 & 67 & 1 & 1 \\
\hline Primary education & 49 & 44 & $\begin{array}{l}1.554 \\
(.896,2.696)^{*}\end{array}$ & $1.745(.966,3.151)$ \\
\hline $\begin{array}{l}\text { High School and } \\
\text { above }\end{array}$ & 221 & 123 & $2.509(1.629,3.86)$ & $2.15(1.359,3.403)^{\star \star}$ \\
\hline \multicolumn{5}{|l|}{ Mode of delivery } \\
\hline Spontaneous delivery & 233 & 187 & 1 & 1 \\
\hline Caesarean delivery & 37 & 18 & $1.65(.91,2.992)$ & $1.543(.81,2.942)$ \\
\hline Instrumental delivery & 48 & 29 & $1.328(.806,2.189)$ & $1.241(.723,2.131)$ \\
\hline \multicolumn{5}{|c|}{ Ever discussed with their husband about FP } \\
\hline No & 152 & 163 & 1 & 1 \\
\hline Yes & 166 & 71 & $2.507(1.758,3.576)$ & $2.152(1.478,3.135)^{\star \star}$ \\
\hline \multicolumn{5}{|l|}{ Attend ANC } \\
\hline No & 5 & 8 & 1 & 1 \\
\hline Yes & 313 & 226 & $2.216(.716,6.862)$ & $.987(.304,3.207)$ \\
\hline \multicolumn{5}{|l|}{ Attend PNC } \\
\hline No & 121 & 117 & 1 & 1 \\
\hline Yes & 197 & 117 & $1.628(1.157,2.29)$ & $1.186(.813,1.729)$ \\
\hline \multicolumn{5}{|c|}{ After delivery of the last child do you get information about FP } \\
\hline No & 180 & 162 & 1 & 1 \\
\hline Yes & 138 & 72 & $1.725(1.209,2.461)$ & $1.42(.964,2.092)$ \\
\hline
\end{tabular}




\begin{tabular}{|c|c|c|c|c|}
\hline Variables & Knowledge & & COR $(95 \% \mathrm{Cl})$ & AOR $(95 \% \mathrm{Cl})$ \\
\hline \multicolumn{5}{|c|}{ History of modern contraceptive before delivery of last child } \\
\hline No & 44 & 53 & 1 & 1 \\
\hline Yes & 274 & 181 & $1.823(1.172,2.836)$ & $1.87(1.154,3.03)^{\star \star *}$ \\
\hline
\end{tabular}

\section{Factors Associated with Attitude of Postpartum Mothers on Modern Contraceptive Methods}

Multivariable regression analysis revealed that maternal age of 25-34 [AOR: 0.42 (95\% Cl: .27, .65)], having prior information about modern contraceptive methods before delivery of the last child [AOR:

1.733 (95\% Cl: 1.19, 2.53)] and knowledge of the mother [AOR: 3.493 (95\% Cl: 2.37, 5.15)] were found to be significantly associated with the attitude of postpartum mothers about modern contraceptives (Table 6). 
Table 6

Bivariable and Multivariable Logistic Regression Analysis of Factors Associated with Attitude of Postpartum Mothers about Modern Contraceptives in Debre Tabor Town, South Gondar Zone, Northwest Ethiopia, $2018(\mathrm{n}=552)$

\begin{tabular}{|c|c|c|c|c|}
\hline \multirow[t]{2}{*}{ Variables } & \multicolumn{2}{|l|}{ Attitude } & \multirow[t]{2}{*}{ COR $(95 \% \mathrm{Cl})$} & \multirow[t]{2}{*}{ AOR $(95 \% \mathrm{Cl}$} \\
\hline & $\begin{array}{l}\text { Favorable } \\
\text { attitude }\end{array}$ & $\begin{array}{l}\text { Un-favorable } \\
\text { attitude }\end{array}$ & & \\
\hline \multicolumn{5}{|l|}{ Age } \\
\hline $18-24$ & 68 & 64 & & \\
\hline $25-34$ & 127 & 226 & $.529(.353, .793)$ & $.42(.27,652) \star \star$ \\
\hline $35-45$ & 29 & 38 & $.718(.397,1.298)$ & $.579(.308,1.089)$ \\
\hline \multicolumn{5}{|c|}{ Ever discussed with their husband about FP } \\
\hline No & 113 & 202 & 1 & 1 \\
\hline Yes & 111 & 126 & $1.575(1.117,2.221) *$ & $1.224(.841,1.783)$ \\
\hline \multicolumn{5}{|l|}{ Attend PNC } \\
\hline No & 80 & 158 & 1 & 1 \\
\hline Yes & 144 & 170 & $1.673(1.18,2.37)^{\star \star}$ & $1.451(.986,2.136)$ \\
\hline \multicolumn{5}{|c|}{ Before delivery of the last child do you get information about FP } \\
\hline No & 106 & 204 & 1 & \\
\hline Yes & 118 & 124 & $1.83(1.298,2.584)^{\star \star}$ & $1.733(1.19,2.525)^{\star \star}$ \\
\hline \multicolumn{5}{|c|}{ After delivery of the last child do you get information about FP } \\
\hline No & 120 & 222 & 1 & 1 \\
\hline Yes & 104 & 106 & $1.82(1.279,2.576)^{\star \star}$ & $1.357(.904,2.037)$ \\
\hline \multicolumn{5}{|c|}{ History of modern contraceptive utilization before delivery of the last child } \\
\hline No & 44 & 53 & 1 & 1 \\
\hline Yes & 274 & 181 & $1.823(1.172,2.836)$ & $.91(.556,1.491)$ \\
\hline \multicolumn{5}{|l|}{ Knowledge } \\
\hline $\begin{array}{l}\text { Poor } \\
\text { knowledge }\end{array}$ & 59 & 175 & 1 & 1 \\
\hline $\begin{array}{l}\text { Good } \\
\text { knowledge }\end{array}$ & 165 & 153 & $3.199(2.214-4.62)$ & $3.493(2.371,5.147)^{\star \star}$ \\
\hline
\end{tabular}




\section{Discussion}

Improving women's knowledge and attitude towards modern contraceptive methods is indispensable to enhance the practice of postpartum contraceptives. This study assessed women's knowledge and attitudes of postpartum modern contraceptive methods in Debre Tabor town.

Accordingly, this study found that the overall knowledge of women on postpartum modern contraceptive methods was $57.6 \%$ which is in line with a study conducted in northwest Ethiopia (11). However, the finding of this study was lower than a study conducted in Eastern Ethiopia (12). The disparity may be the difference in the study period in which the previous study was done on postpartum mothers at the end of twelve months which may have repetitive contact in $\mathrm{MNCH}$ services that increase the chance to get more information about family planning methods and other maternal health services.

The level of mother's good knowledge of postpartum modern contraceptives in this study was higher than the study conducted in Northwest Ethiopia (16). This may be due to the difference in the study subjects which means the previous study was done in all reproductive-aged mothers but this study was conducted on postpartum mothers which may be an opportunity to get information from $\mathrm{MNCH}$ contact of mothers.

Women's knowledge of at least one postpartum modern contraceptive method was $87.3 \%$ with $95 \% \mathrm{Cl}$ (84.4\%, 90\%). The finding of this study was lower than a study conducted at Adama Hospital, Oromia region, Ethiopia (13). One explanation might be due to the study design which was an institutional-based study design but this study was a community-based study design. Another explanation might be the study period and the study population in which the information we gathered. Our study was done on the extended postpartum period but that study in Oromia region was done on the immediate postpartum period which may increase mothers to get information about family planning methods in the maternity ward. However, the finding of this study is higher than a study which was done in northern India (17). This may be due to socio-demographic and cultural differences in the study population.

In this study, the educational status of women was found to be a predictor of mother's knowledge of postpartum contraceptive methods. Those mothers who attend primary and above education were 2.15 times more likely to have good knowledge of postpartum modern contraceptive methods compared to those women who didn't attend formal education. This study is supported by a study conducted in Northern India (17). This could be due to educated mothers may get information about contraceptive methods from different sources like by attending maternal health services and other reproductive issues and able to retain the information they attained.

The odds of having adequate knowledge of postpartum modern contraceptive methods among women who have ever discussed with their husbands about contraceptive methods were two times higher compared to those who had poor knowledge. This could be justified as a discussion for contraceptive methods with partners that may increase the chance to share different information about modern 
contraceptive methods and get the freedom to do any maternal and reproductive health services. This in turn enhances their knowledge of contraceptive methods.

History of modern contraceptive utilization before the delivery of the last child was also another important factor for mothers' knowledge of modern contraceptive methods. Those mothers who have ever used modern contraceptive methods before the delivery of the last child were nearly two times more likely to have had good knowledge of postpartum contraceptive methods compared to their counterparts. This could be due to women who have ever used contraceptive methods may discuss and share ideas about different contraceptive methods with health professionals.

The current study found that the attitude of mothers towards modern contraceptive methods was $40.6 \%$ with 95\% $\mathrm{Cl}(36.8,44.6)$ which is lower than a study done in Eastern Ethiopia and Khatamendu, Nepal $(16,18)$. These differences might be due to the dissimilarity in socio-demographic characteristics. The other difference from a study conducted in Nepal might be the result of the tool we used to measure the outcome variable; in this study, the questions were prepared on five Likert scales (strongly disagree, disagree, neutral, agree and strongly agree) and also might be the difference in study design.

Mothers in the age groups of $25-34$ were $58 \%$ less likely to have had a favorable attitude compared to mothers in the age group of 18-24 years old.

Our study declared that having prior information about contraceptive methods before the delivery of the most recent child was 1.73 times more likely to have had a positive attitude towards postpartum contraceptive method utilization. This may be due to having prior information that may help the women to have sufficient knowledge and outweigh the prone and cons of not using contraceptive methods thereby developing a favorable attitude through time.

Lastly, adequate knowledge of postpartum modern contraceptive methods is found to be a significant factor associated with the mother's attitude towards postpartum modern contraceptive method utilization. Those women who had good knowledge were 3.49 times more likely to have had a favorable attitude towards postpartum modern contraceptive methods. This is because knowledge is an entry point for any behavior and health service-related practices. Also, knowledgeable women have better retaining and comprehension skills about maternal and reproductive health-related activities compared to women who have poor knowledge.

\section{Conclusion}

More than half of the respondents had good knowledge about modern contraceptive methods. But, mother's favorable attitude towards its utilization is lower compared to other studies. Mother's higher educational status, ever discussed with their husband about contraceptive methods, and ever used contraceptive methods were the factors significantly associated with knowledge of postpartum modern contraceptive methods. Furthermore, adequate knowledge of postpartum modern contraceptive methods, age group of 25-34 years old and having prior information about contraceptive methods were the factors 
that significantly associated with the attitude of women. Integrated work is necessary to build up women's access to education, to get information all through the maternal continuum of care, and encourage couples to have a free discussion regarding contraceptive methods and other health-related activities.

\section{Abbreviations}

ANC

antenatal care, AOR:adjusted odds ratio, Cl:confidence interval, COC:combined oral contraceptives, COR:crud odds ratio, FP:family planning, IUCD:intra-uterine contraceptive device, $\mathrm{MCH}$ :maternal and child health, PNC:postnatal care, PPFP:postpartum family planning, SPSS:statistical package for social scientists, WHO:world health organization

\section{Declarations}

\section{Ethics Approval and Consent to Participate}

This study was conducted under the declaration of Helsinki. Ethical clearance was obtained from the School of Midwifery (MIDW/10/489/2018) under the delegation from the Ethical Review Board of the University of Gondar. Verbal and written informed consent was obtained from each study participants after informing the objective of the study. In the consent, statements about the potential risks, benefits, and confidentiality were included and explained.

Consent for publication: Not applicable

\section{Data Sharing Statements}

The datasets collected and analyzed for this study are available from the corresponding author and can be attained on a reasonable request

Competing Interest: The authors declare that they have no competing interests.

\section{Funding Statement}

The funding source of this research was the University of Gondar and the University has no role in design, data collection, analysis, decision to publish as well as preparation of the manuscript.

\section{Authors' Contributions}

All the authors had significant involvement in the conception and designing the study, acquisition of data, analysis and interpretation of data, took part in drafting the article, revising the article, gave final approval of the version to be published, have agreed on the journal to which the article has been submitted and agree to be accountable for all aspects of the work. 


\section{Acknowledgments}

We would like to thank the University of Gondar for the financial support. We are also very thankful to the managing bodies of Debre Tabor town administrators. We deeply appreciate the data collectors and the study subjects.

\section{References}

1. Kesetebirhan A. National Guideline for Family Planning Services in Ethiopia. Federal Democratic Republic of Ethiopia, Ministry of Health. 2011.

2. Quereishi MJ, Mathew AK, Sinha A. Knowledge, attitude and practice of family planning methods among the rural females of Bagbahara block Mahasamund district in Chhattishgarh State, India. Glob J Med Public Heal. 2017;6(2):1-7.

3. Commission NP. The 2017 Voluntary National Reviews on SDGs of Ethiopia: Government Commitments, National Ownership and PerformanceTrends Federal Democratic Republic of Ethiopia. 2017.

4. Salisbury P, Hall L, Kulkus S, Paw MK, Tun NW, Min AM, et al. Family planning knowledge, attitudes and practices in refugee and migrant pregnant and post-partum women on the Thailand-Myanmar border-a mixed methods study. Reproductive health. 2016;13(1):94.

5. EDHS E. demographic and health survey 2016: key indicators report. The DHS Program ICF. 2016.

6. EDHS. ETHIOPIA Demographic and Health Survey 2016.

7. Smith R, Ashford L, Gribble J, Clifton D. Family planning saves lives. 2009.

8. Rutstein SO, Winter R. Contraception needed to avoid high-fertility-risk births, and maternal and child deaths that would be averted: ICF International; 2015.

9. Berta M, Feleke A, Abate T, Worku T, Gebrecherkos T. Utilization and associated factors of modern contraceptives during extended postpartum period among women who gave birth in the last 12 months in Gondar Town, northwest Ethiopia. Ethiopian Journal of Health Sciences. 2018;28(2):20716.

10. Abraha TH, Teferra AS, Gelagay AA. Postpartum modern contraceptive use in northern Ethiopia: prevalence and associated factors. Epidemiology and health. 2017;39.

11. Gizaw W, Zewdu F, Abuhay M, Bayu H. Extended Postpartum Modern Contraceptive Utilization and Associated Factors among Women in Gozamen District, East Gojam Zone, Northwest Ethiopia, 2014. Insights Reprod Med. 2017;1(2):8.

12. At N, D G, G T. Postpartum Family Planning Utilization and Associated Factors among Women who Gave Birth in the Past 12 Months, Kebribeyah Town, Somali Region, Eastern Ethiopia. Journal of Women's Health Care. 2016;05(06).

13. Ngusu M, Wondafrash B, Segni H, Gurmessa A. Knowledge, Attitude and Practice of Family Planning Methods Among Laboring Mothers in Adama Hospital, Oromia Region, Ethiopia. J Womens Health, 
Issues Care 4. 2015;3:2.

14. Federal Democratic Republic of Ethiopia Population Census Commission. Summary and Statistical report of the 2007 population and housing census:population size by age and sex. United Nations Population Fund. 2008.

15. Gebremariam A, Gebremariam H. Contraceptive use among lactating women in Ganta-Afeshum District, Eastern Tigray, Northern Ethiopia, 2015: a cross sectional study. BMC pregnancy and childbirth. 2017;17(1):421.

16. Kasa AS, Tarekegn M, Embiale N. Knowledge, attitude and practice towards family planning among reproductive age women in a resource limited settings of Northwest Ethiopia. BMC research notes. 2018;11(1):577.

17. Nath J, Islam F. A study on the knowledge, attitude and practice about contraception in postpartum women of North India. Parity. 2015;35(45):45.

18. Bajracharya A. Knowledge, attitude and practice of contraception among postpartum women attending Kathmandu Medical College Teaching Hospital. Kathmandu University Medical Journal. 2015;13(4):292-7.

\section{Figures}

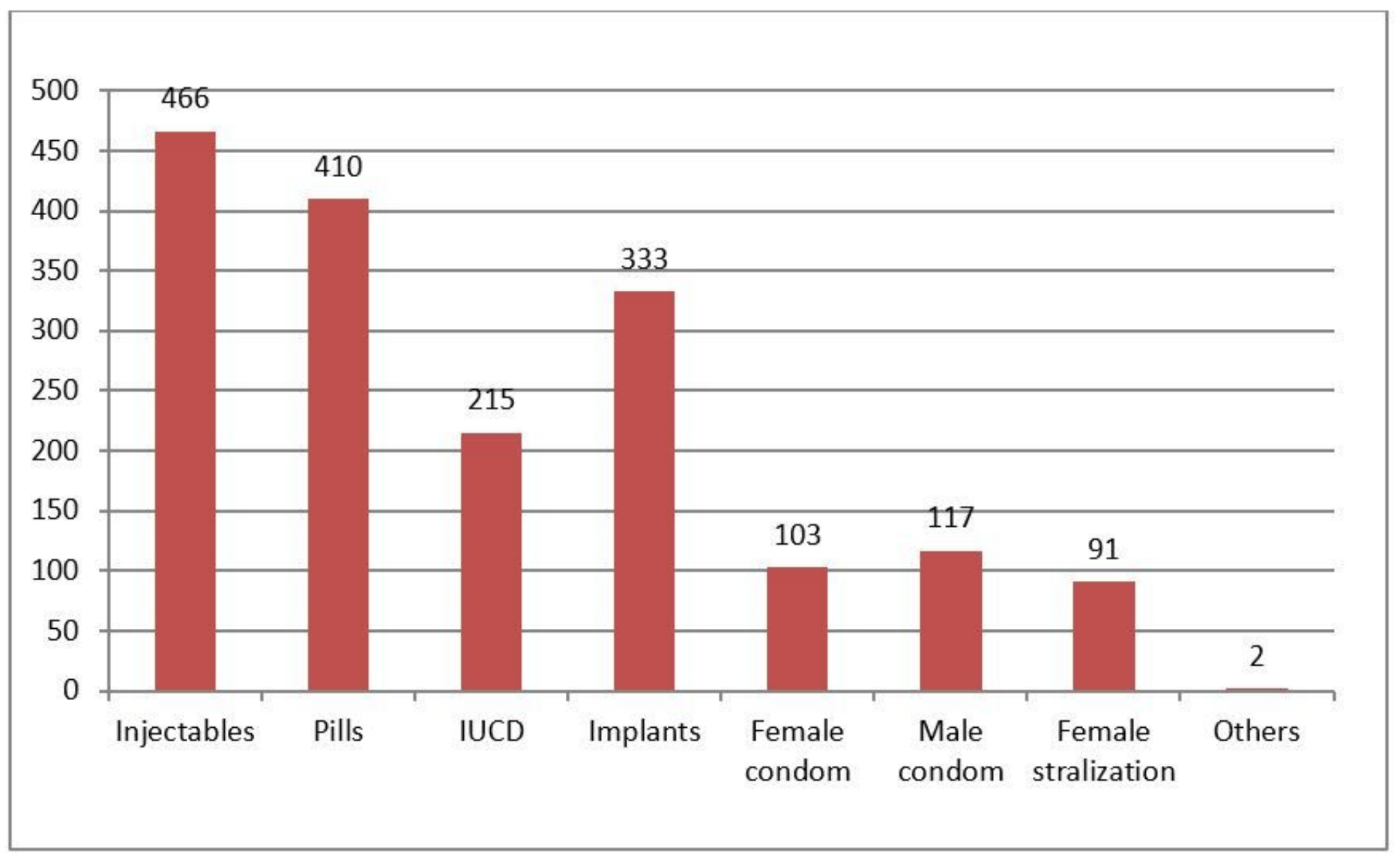




\section{Figure 1}

Type of contraceptives known by postpartum mothers in Debra Tabor town, South Gondar zone, Northwest Ethiopia 2018. 